\title{
Análisis de crecimiento del cultivo de balsamina Momordica charantia L. en semillero
}

Growth analysis of the bitter gourd Momordica charantia L. crop in nursery phase

\section{Fernando Vicente Barraza A. ${ }^{1}$; Orlando Edmundo Benavides B. ${ }^{2}$; Yonger Tamayo $\mathrm{A}^{3}$}

1 Profesor. I.A., Ph.D. Universidad de Córdoba, Montería.Colombia. barraza@fca.edu.co

2 Profesor. I.A. M.Sc. Universidad de Nariño, Pasto. Colombia. orlando.benavides2@gmail.com

3 Profesor. I.A. M.Sc., Universidad de Guantánamo, Cuba. yongertamayo@cug.co.cu

\section{RESUMEN}

Esta investigación se realizó en el campo experimental de cultivos de hortalizas de la Universidad de Córdoba, Colombia, con el objetivo de analizar el crecimiento de plántulas de balsamina Momordica charantia L. obtenidas a partir de semillas sometidas a tratamientos pre-siembra, ya que su cubierta es demasiado gruesa y provoca retraso en el crecimiento. Se utilizó el diseño completamente al azar. Las semillas después de extraídas de la planta se sometieron a los siguientes tratamientos: T1 (Testigo), T2 (semillas lavadas a temperatura ambiente (TA) y T3 (semillas remojadas en agua a TA durante 24 horas). Las variables estudiadas fueron área foliar (AF), materia seca de hojas (MSH), materia seca total (MST), tasa absoluta de crecimiento (TAC), tasa relativa de crecimiento (TRC), tasa de asimilación neta (TAN), área foliar específica (AFE), índice de área foliar (IAF), relación de área foliar (RAF) y relación de peso foliar (RPF). Se encontró una trayectoria sigmoide, ajustada al modelo logístico en AF, MSH y MST. Respecto a AF y MST, el tratamiento tres superó a los demás a los 15, 21 y 24 días después de la siembra (dds). Con relación a TAC, AFE, RAF y RPF se observó una gráfica de distribución normal. Para T3 hubo mayor TAC a los 16 dds $\left(0,023\right.$ g.día $\left.{ }^{-1}\right)$. Respecto a TAN y TRC, se observó comportamiento decreciente en el tiempo. El IAF fue mayor para T3 durante toda la fase de semillero.

Palabras clave: Remojo de semillas, materia seca, área foliar. 


\begin{abstract}
An experimental field research study was conducted with vegetable crops at the University of Cordoba, Colombia, with the objective of analize the growth of bitter gourd seedlings Momordica charantia L obtained from seeds under pre-planting treatments, given the presence of a very thick seed coat that causes growth delay. A completely randomized design was used. After extraction from the plant, the seeds were subjected to the following treatments: T1 (control), T2 (seeds rinsed with ambient temperature water (TA)), and T3 (seeds submerged in ambient temperature water during 24 hours). The study variables were leaf area (AF), dry leaf matter (MSH), total dry matter (MST), absolute growth rate (TAC), relative growth rate (TRC), net assimilation rate (TAN), specific leaf area (AFE), leaf area index (IAF), leaf area ratio (RAF), and leaf weight ratio (RPF). A sigmoidal trajectory was found, which adjusted to the logistic model in AF, $\mathrm{MSH}$, and MST. In regards to AF and MST, treatment 3 exceeded the other treatments at days 15, 21, and 24 after planting (dds). Regarding TAC, AFE, RAF, and RPF, a normal distribution graph was observed. A higher TAC was obtained for T3 at day 16 (0.023 g. day $^{-1}$ ). For TAN and TRC, a declining behavior was observed throughout time. The IAF was highest for $\mathrm{T} 3$ during the entire nursery phase.
\end{abstract}

Keywords: Seed submergence, dry matter, leaf area.

\section{INTRODUCCIÓN}

Como balsamina, melón amargo, pera de bálsamo, cundeamor, tomaco, calabaza africana, pepino africano, entre otros, se conoce en el mundo a la hortaliza tropical M. charantia (Zong et al., 1995; Mia et al., 2014). Sus hojas se utilizan como verdura y en preparación de curry. Los frutos inmaduros se consumen crudos, hervidos, fritos o encurtidos. Las semillas tostadas y molidas sirven de condimento y pueden consumirse hervidas y fritas. También son comestibles las raíces, flores y arilo de las semillas maduras (Shahadat et al., 2008; Thakur et al., 2009).

En el país hace parte de las especies de "Plantas Útiles de Colombia" (Pérez-Arbeláez, 1994) y es considerada además arvense de cultivos. En la
Costa Atlántica se consumen sus arilos de sabor dulce, que también son apetecidos por las serpientes de acuerdo con la tradición oral campesina (Barraza, 2015), quien reporta usos ancestrales como antitumoral, abortiva, emenagogo, febrífugo, en emplastos y cataplasmas externos contra hemorroides, afecciones cutáneas, prurito, aftas y quemaduras. El Instituto Nacional de Vigilancia de Medicamentos y Alimentos (INVIMA) aprueba su uso como hipoglucemiante (Fonnegra y Jiménez, 2007).

Debido al potencial bio-farmacológico y alimenticio de la balsamina, es una planta que amerita ser investigada desde el nivel básico, incluida en los programas de seguridad nutracéutica y explotada comercialmente como cultivo promisorio (Thakur et al., 2009). 
Uno de los principales tópicos de investigación básica que se justifica abordar dentro del estudio de $M$. charantia, es el análisis de crecimiento del cultivo, inicialmente en etapa de semillero, ya que es un proceso bastante rápido y de acuerdo con el manejo agronómico efectuado, hay influencia posterior en el rendimiento de frutos y su composición química (Fohs et al., 2014; Şavşatliy y Seyis, 2014).

A través del análisis de crecimiento, puede darse una explicación holística e integradora de cómo crece la planta y cómo funcionan sus procesos fisiológicos, a medida que se dan algunos cambios estructurales y bioquímicos específicos, utilizando métodos cuantitativos para describir e interpretar los cambios en tamaño, peso, volumen, área y contenidos de componentes de la planta, que ocurren de acuerdo con los patrones de división y diferenciación celular y que tienen relación con el ambiente, ya sea bajo condiciones naturales o controladas (Hunt et al., 2002).

Otro aspecto importante sobre el análisis de crecimiento vegetal, es que aporta bases técnicas fundamentales para el manejo de los cultivos, con el fin de obtener las mejores características agronómicas en su manejo, las cuales se pueden estudiar a partir de medidas directas del crecimiento (Evans, 1972; Hunt, 1982 y Clavijo, 1989).

El crecimiento vegetal, como la mayoría de los procesos biológicos, es de naturaleza no lineal; por ello, en el análisis del crecimiento se requiere la utilización de modelos matemáticos no lineales para su descripción e interpretación. Así, una de las ventajas fundamentales con la que cuenta en la actualidad el análisis del crecimiento es el uso de ordenadores potentes y la existencia de programas para el ajuste de modelos no lineales, a través de los cuales se puede obtener información sobre los procesos fisiológicos de la planta, entre los que se destacan el transporte y asignación de carbono (Calvo et al. 1994; Prusinkiewicz, 2004).

Además de lo anterior, mediante el uso de medidas cuantitativas del crecimiento, se pueden estimar medidas indirectas del crecimiento como la tasa absoluta de crecimiento (TAC), tasa relativa de crecimiento (TRC), tasa de asimilación neta (TAN), área foliar específica (AFE), índice de área foliar (IAF), razón de área foliar (RAF) y razón de peso foliar (RPF), entre otros (Gardner et al., 1990; Hunt, 1990 y Causton, 1994).

En vista de la importancia del análisis de crecimiento sobre el estudio del comportamiento fisiológico de los cultivos y su relación con la productividad primaria (Criollo y García, 2009), el objetivo de la presente investigación fue realizar el análisis de crecimiento de $M$. charantia en fase de semillero, a través de las medidas directas de área foliar (sistema asimilatorio), materia seca de hojas y materia seca total. Dichas variables, se sometieron a un proceso de regresión no lineal y se ajustaron al modelo logístico, el cual describe con mucha precisión el comportamiento del crecimiento vegetal y permite a través del cálculo la obtención de medidas indirectas del crecimiento como TAC, TRC, TAN, AFE, IAF, RAF y RPF, que pueden explicar la relación entre el sistema asimilatorio, producción de biomasa 
y transporte de asimilados (Borrego et al., 2000; Báez et al., 2002).

\section{MATERIALES Y MÉTODOS}

La investigación se realizó en la Universidad de Córdoba, Colombia, entre febrero 6 y abril 10 de 2014, periodo que tuvo un promedio mensual de temperatura de $28,7^{\circ} \mathrm{C}$, humedad relativa de $76 \%$ y precipitación de 90,7 mm. Se estableció un bioensayo para el análisis de crecimiento de plántulas del cultivo de $M$. charantia en fase de semillero, en el Campo Experimental de Cultivos de Hortalizas de la Facultad de Ciencias Agrícolas. Las semillas sembradas se colectaron de cultivos del genotipo Criollo en la Universidad de Córdoba. Se utilizó diseño Completamente al Azar con tres tratamientos (Tn) y cuatro repeticiones. Dichos tratamientos fueron los siguientes: T1 (Testigo), T2 (semillas lavadas con agua a temperatura ambiente (TA)) y T3 (semillas remojadas en agua a temperatura ambiente (TA) durante 24 horas).

En cada repetición se sembraron 25 semillas a $0,5 \mathrm{~cm}$ de profundidad, para un total de $100 \mathrm{se}-$ millas por tratamiento y 300 semillas para el bioensayo. Cada repetición, ocupó 0,09 m², con separación de 0,15 m entre repeticiones, para una superficie total de $2,0 \mathrm{~m}^{2}$.

Desde los 7 hasta los 30 días después de la siembra, se tomaron datos cada tres días de las variables área foliar $\left(A F\right.$, expresada en $\left.\mathrm{cm}^{2}\right)$, materia seca de hojas (MSh, expresada en gramos) y materia seca total (MSt, expresada en gramos).
Para materia seca de hojas y total, se sometió el material vegetal a secado en horno eléctrico Memmert ${ }^{\circledR} \mathrm{U} 10$ a $85{ }^{\circ} \mathrm{C}$ hasta obtención de peso constante, tomado con balanza electrónica de precisión Ohaus ${ }^{\circledR}$ Explorer EX1103. El área foliar se obtuvo mediante procesamiento digital de las hojas con el software DDA (Ferreira et al., 2009) acoplado a scanner HP ScanJet ${ }^{\circledR} 3400$ C.

Los datos de área foliar, materia seca de hojas y materia seca total, fueron sometidos a análisis de regresión no lineal con el procedimiento PROC NLIN mediante el software SAS versión 9.1.3 (SAS Institute, 2008) y se ajustaron mediante la utilización del mismo software con el modelo logístico: $y=\frac{A}{\left(1+B \cdot e^{-C \cdot x}\right)}$, donde: $\mathrm{y}=$ variable respuesta (área foliar, materia seca de hojas, materia seca total, en función de $x$ ); $x$ = tiempo (ddt); $A=$ asíntota, valor de $\mathrm{y}$ cuando $x$ tiende a infinito; $B \cdot e^{-c x}=$ factor de amortiguamiento, donde: $B=$ corresponde a un factor de amplitud, no tiene significado biológico y solo toma lugar en el tiempo inicial, cuando $x=0 \mathrm{y}$ $\mathrm{C}=$ factor de estabilidad relacionado con el valor de $x$, e número de Euler.

Para calcular los valores instantáneos de las medidas indirectas del crecimiento a partir de los datos de área foliar, materia seca de hojas y materia seca total, se utilizaron las ecuaciones presentadas en la Tabla 1 (Gardner et al., 1990).

A las variables estudiadas se sometieron al Análisis de Varianza y Pruebas de Comparación de Medias de Tukey $(p \leq 0,05)$ mediante el software SAS (PROC ANDEVA). 
Tabla 1. Ecuaciones para cálculo de índices de eficiencia fotosintética de $M$. charantia.

\begin{tabular}{lccl}
\hline \multicolumn{1}{c}{ Índice } & Símbolo & Valor instantáneo & \multicolumn{1}{c}{ Unidades } \\
\hline Tasa absoluta de crecimiento & TAC & $\frac{d M S t}{d t}$ & $\mathrm{~g} \cdot \mathrm{dí}^{-1}$ \\
Tasa relativa de crecimiento & TRC & $\frac{1}{M S t} \cdot \frac{d M S t}{d t}$ & $\mathrm{~g} \cdot \mathrm{g}^{-1} \cdot \mathrm{día}^{-1}$ \\
Tasa de asimilación neta & TAN & $\frac{1}{A F} \cdot \frac{d M S t}{d t}$ & $\mathrm{~g} \cdot \mathrm{cm}^{-2} \cdot \mathrm{día}^{-1}$ \\
Área foliar específica & AFE & $\frac{A F}{M S h}$ & $\mathrm{~cm}^{2} \cdot \mathrm{g}^{-1}$ \\
Relación de área foliar & RAF & $\frac{A F}{M S t}$ & $\mathrm{~cm}^{-2} \cdot \mathrm{g}^{-1}$ \\
Índice de área foliar & IAF & $\frac{A F}{S t}$ & Sin dimensiones \\
Relación de peso foliar & RPF & $\frac{M S h}{M S t}$ & $\%$ \\
\hline
\end{tabular}

MSt: materia seca total, AF: área foliar, MSh: materia seca de hojas, St: superficie de terreno.

\section{RESULTADOS Y DISCUSIÓN}

Área foliar, materia seca de hojas y materia seca total. Para estas variables se presentó un comportamiento gráfico de curva simple sigmoide (Figuras 1, 2 y 3), el cual ha sido reportado también para otras variables del crecimiento en el fruto de $M$. charantia como longitud, diámetro y acumulación de materia seca con el cultivar Moon Shine (Kuo et al., 2012) y en fase de semillero de Solanum melongena L. (Barraza, 2013) y se ajustaron al modelo logístico (Tabla. 2).

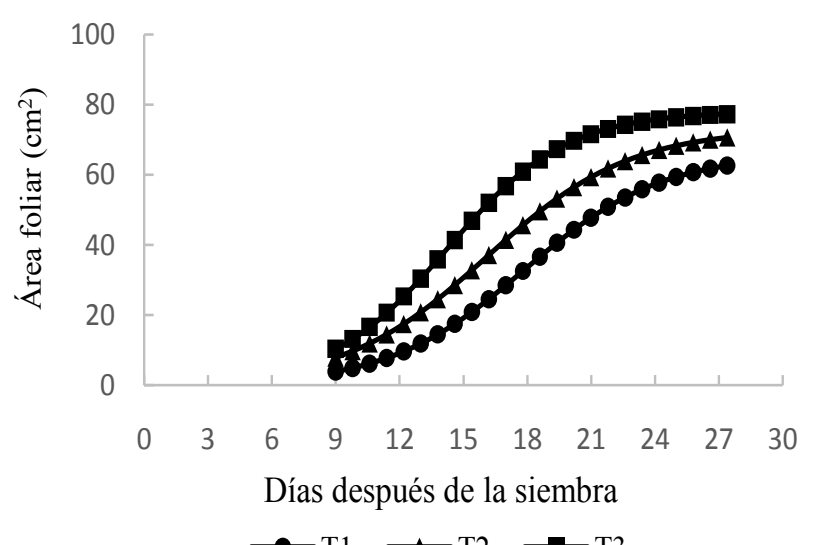

Figura 1. Área foliar de M. charantia en fase de semillero. T1 (Testigo), T2 (semillas lavadas con agua) y T3: (semillas remojadas en agua a TA durante 24 horas). Montería, Colombia, 2014.

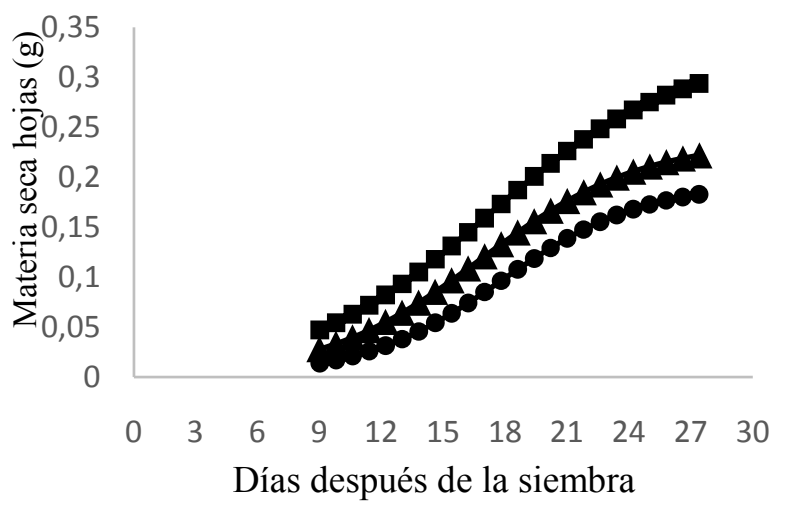

Figura 2. Acumulación de materia seca de hojas de M. charantia en fase de semillero. T1 (Testigo), T2 (semillas lavadas con agua a TA) y T3: (semillas remojadas en agua TA durante 24 horas). Montería, Colombia, 2014.

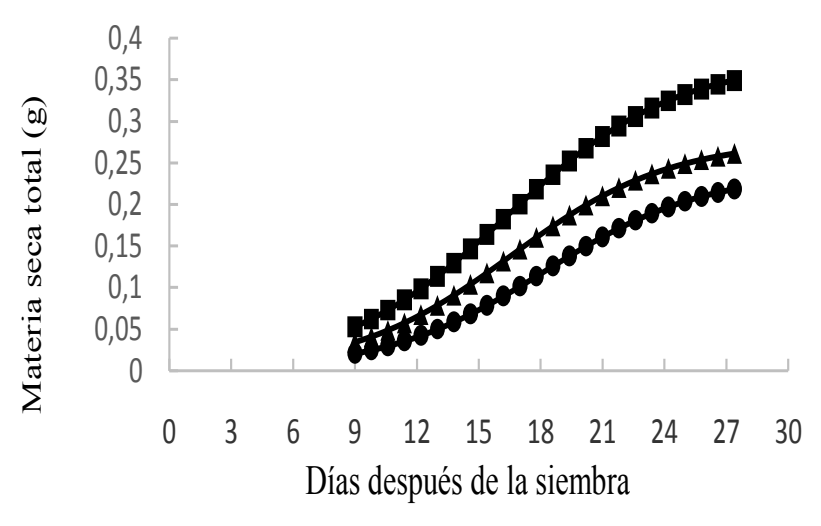$$
\rightarrow-\mathrm{T} 1 \longrightarrow \mathrm{T} 2 \rightarrow-\mathrm{T} 3
$$

Figura 3. Acumulación de materia seca total de M. charantia en fase de semillero. T1 (Testigo), T2 (semillas lavadas con agua a TA) y T3: (semillas remojadas en agua TA durante 24 horas). Montería, Colombia, 2014. 
Tabla 2. Modelos logísticos para estimar crecimiento en área foliar, materia seca de hojas y materia seca total de plántulas de $M$. charantia en fase de semillero.

Montería, Colombia, 2014.

\begin{tabular}{|c|c|c|}
\hline \multicolumn{2}{|c|}{ Tratamiento } & $\mathrm{R}^{2}$ \\
\hline \multicolumn{3}{|c|}{ Área foliar $\left(\mathrm{cm}^{2}\right)$} \\
\hline $\mathrm{T} 1$ & $\mathrm{y}=65,9947 /\left(1+254,6 \mathrm{e}^{-0,310^{*} \mathrm{dds}}\right)$ & 0,99 \\
\hline $\mathrm{T} 2$ & $\mathrm{y}=73,1107 /\left(1+121,0 \mathrm{e}^{-0,298^{*} \mathrm{dds}}\right)$ & 0,99 \\
\hline $\mathrm{T} 3$ & $\mathrm{y}=78,0637 /\left(1+163,3 \mathrm{e}^{-0,357^{*} \mathrm{dds}}\right)$ & 0,96 \\
\hline \multicolumn{3}{|c|}{ Materia seca de hojas (g) } \\
\hline T1 & $\mathrm{y}=0,1941 /\left(1+185,7 \mathrm{e}^{-0,292^{*} \mathrm{dds}}\right)$ & 0,99 \\
\hline $\mathrm{T} 2$ & $\mathrm{y}=0,2364 /\left(1+75,4 \mathrm{e}^{-0,257^{*} \mathrm{dds}}\right)$ & 0,99 \\
\hline $\mathrm{T} 3$ & $\mathrm{y}=0,268 /\left(1+41,7 \mathrm{e}^{-0,216^{*} \mathrm{dds}}\right)$ & 1,00 \\
\hline \multicolumn{3}{|c|}{ Materia seca total $(\mathrm{g})$} \\
\hline T1 & $\mathrm{y}=0,2395 /\left(1+105,2 \mathrm{e}^{-0,256^{*} \mathrm{dds}}\right)$ & 1,00 \\
\hline $\mathrm{T} 2$ & $\mathrm{y}=0,2771 /\left(1+73,3 \mathrm{e}^{-0,259^{*} \mathrm{dds}}\right)$ & 0,98 \\
\hline $\mathrm{T} 3$ & $\mathrm{y}=0,3729 /\left(1+54,8 \mathrm{e}^{-0,244^{*} \mathrm{dds}}\right)$ & 0,99 \\
\hline
\end{tabular}

Para el tratamiento tres, las variables área foliar, materia seca de hojas y materia seca total tuvieron un comportamiento estadísticamente superior a los tratamientos uno y dos, así, para área foliar a los 15, 21 y 24 dds, para materia seca de hojas, a los 15 y 24 días despues de la siembra, y para materia seca total, a los 15, 21 y 24 dds (Tabla 3).

De acuerdo con lo anterior, se observó que el remojo de semillas en agua TA durante 24 horas (T3) mejoró el proceso de crecimiento de las plantas durante su fase de semillero, ya que en dicho cultivo, uno de los principales inconvenientes para la emergencia en campo lo constituye el retraso en el crecimiento que provoca el elevado grosor de la cubierta de las semillas (Saleem et al., 2014) y concuerda con los resultados obtenidos con dicha técnica de presiembra por Ashrafuzzaman et al. (2010) quienes además indican que ofrece ventajas adicionales, como la mejora en el establecimiento del cultivo, uso de menor cantidad de semillas y la posibilidad de descartar semillas muertas y de bajo vigor antes de la siembra.

Tabla 3. Área foliar, materia seca de hojas y acumulación de materia seca total de $M$. charantia en fase de semillero, Montería, Colombia, 2014.

\begin{tabular}{cccc}
\hline \multirow{4}{*}{ dds } & \multicolumn{3}{c}{ Área foliar $\left(\mathrm{cm}^{2}\right)$} \\
\cline { 2 - 4 } & T1 & T2 & T3 \\
\hline 9 & $5,54 \mathrm{a}$ & $12,63 \mathrm{~b}$ & $15,40 \mathrm{~b}$ \\
12 & $8,67 \mathrm{a}$ & $15,38 \mathrm{~b}$ & $19,23 \mathrm{~b}$ \\
15 & $18,85 \mathrm{a}$ & $25,43 \mathrm{a}$ & $44,93 \mathrm{~b}$ \\
18 & $33,47 \mathrm{a}$ & $52,72 \mathrm{~b}$ & $64,59 \mathrm{~b}$ \\
21 & $48,64 \mathrm{a}$ & $57,03 \mathrm{a}$ & $69,15 \mathrm{~b}$ \\
24 & $57,01 \mathrm{a}$ & $66,77 \mathrm{~b}$ & $76,28 \mathrm{c}$ \\
\hline \multirow{4}{*}{ dds } & \multicolumn{4}{c}{ Materia seca de hojas $(\mathrm{g})$} \\
\cline { 2 - 4 } 9 & $0,01 \mathrm{a}$ & $0,04 \mathrm{~b}$ & $0,05 \mathrm{~b}$ \\
9 & $0,03 \mathrm{a}$ & $0,04 \mathrm{~b}$ & $0,06 \mathrm{~b}$ \\
12 & $0,06 \mathrm{a}$ & $0,09 \mathrm{a}$ & $0,15 \mathrm{~b}$ \\
15 & $0,10 \mathrm{a}$ & $0,15 \mathrm{a}$ & $0,17 \mathrm{a}$ \\
18 & $0,13 \mathrm{a}$ & $0,17 \mathrm{a}$ & $0,21 \mathrm{a}$ \\
21 & $0,17 \mathrm{a}$ & $0,21 \mathrm{a}$ & $0,27 \mathrm{~b}$ \\
24 & Acumulación de materia seca total (g) \\
\hline \multirow{4}{*}{ Tds } & \multicolumn{4}{c}{ T1 } & T2 & T3 \\
\cline { 2 - 4 } 9 & $0,03 \mathrm{a}$ & $0,05 \mathrm{a}$ & $0,06 \mathrm{a}$ \\
12 & $0,04 \mathrm{a}$ & $0,06 \mathrm{~b}$ & $0,07 \mathrm{~b}$ \\
15 & $0,07 \mathrm{a}$ & $0,10 \mathrm{a}$ & $0,18 \mathrm{~b}$ \\
18 & $0,12 \mathrm{a}$ & $0,16 \mathrm{a}$ & $0,22 \mathrm{a}$ \\
21 & $0,16 \mathrm{a}$ & $0,22 \mathrm{~b}$ & $0,28 \mathrm{c}$ \\
\hline & $0,19 \mathrm{a}$ & $0,24 \mathrm{a}$ & $0,33 \mathrm{~b}$ \\
\hline
\end{tabular}

dds = días después de la siembra; T1 (Testigo), T2 (semillas lavadas con agua a TA) y T3: (semillas remojadas en agua aTA durante 24 horas). Valores con la misma letra dentro de tratamientos en cada hilera son iguales de acuerdo con la prueba de Tukey $(\mathrm{p} \leq 0,05)$. 
Análisis de crecimiento. La TAC, presentó la forma típica de campana o distribución normal, reportada para varias plantas hortícolas (Ardila et al., 2011). Para todos los tratamientos el crecimiento en términos de velocidad absoluta de acumulación de materia seca fue lento al inicio y fue aumentando a medida que transcurrió el tiempo, a distintas ratas de crecimiento (Figura 4). Para el caso del tratamiento tres, se encontró que la TAC presentó mayores valores durante toda la fase de semillero, con diferencias estadísticas significativas respecto a los demás tratamientos (Tabla 4), lo que implica fisiológicamente mayor capacidad fotosintética de las plantas sometidas a dicho tratamiento para producir nuevos asimilados y refleja también la eficiencia con la que es realizado el proceso (Hunt, 1982). Esto puede estar fundamentado en el hecho de que el remojo en agua favorece el crecimiento inicial, sin riesgos fisiológicos para las semillas (Harris, 2003) y es una respuesta de $M$. charantia a la presión ejercida por el manejo agronómico y la interacción con el ecosistema (Behera et al., 2008 yWang et al., 2010).

Respecto a la TRC, al inicio del cultivo para todos los tratamientos hubo valores altos que

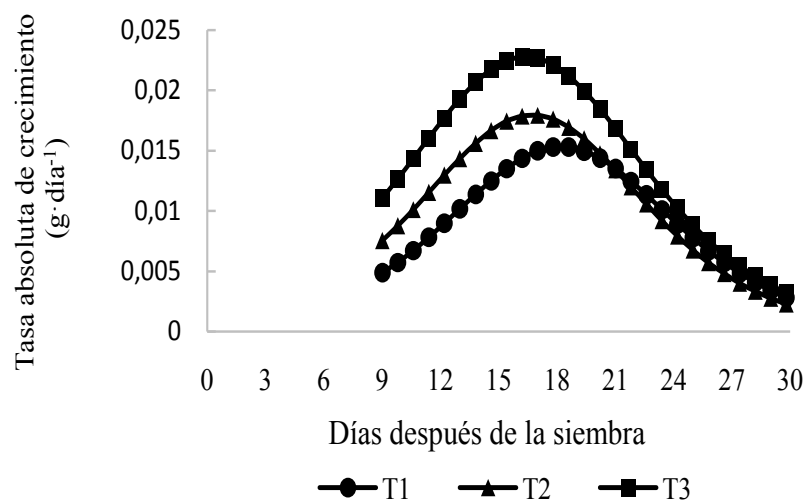

Figura 4. Tasa absoluta de crecimiento de M. charantia en fase de semillero. T1 (Testigo), T2 (semillas lavadas con agua a TA) y T3: (semillas remojadas en agua TA durante 24 horas). Montería, Colombia, 2014. fueron disminuyendo durante el ciclo biológico (Figura 5). Dicho comportamiento ha sido encontrado para Momordica dioica Roxb (Mian et al., 1993) y M. charantia con semillas sometidas a campos eléctricos y magnéticos (Mahajan y Pandey, 2015).

En general, para el tratamiento uno, la TRC tuvo valores más altos con respecto a los demás tratamientos, con diferencias estadísticas significativas (Tabla 4), lo cual implica conceptualmente, que durante el crecimiento y desarrollo integral de la planta, la materia seca producida inicialmente no fue suficiente para seguir produciendo de manera eficiente nueva materia seca que propiciara un crecimiento equilibrado en tamaño de plantas y órganos de interés, lo que en la mayoría de los casos se puede manifestar en menor crecimiento de plántulas y menor longitud de tallo y raíces en semillero, lo que afecta la calidad agronómica y fisiológica del material vegetal para trasplante, aunque la acumulación de materia seca total sea mayor o incluso igual a la de los demás tratamientos (Poorter, 1989; Gardner et al., 1990; Mahajan y Pandey, 2015).

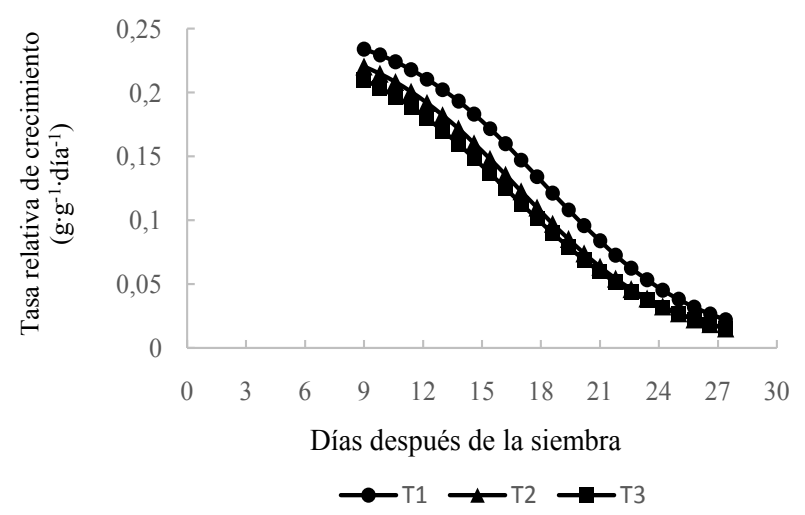

Figura 5. Tasa relativa de crecimiento de $\mathrm{M}$. charantia en fase de semillero. T1 (Testigo), T2 (semillas lavadas con agua aTA) y T3: (semillas remojadas en agua a TA durante 24 horas). Montería, Colombia, 2014. 
Tabla 4. Tasa absoluta de crecimiento, tasa relativa de crecimiento y tasa de asimilación neta de M. Charantia en fase de semillero, Montería, Colombia, 2014.

\begin{tabular}{|c|c|c|c|}
\hline \multirow{2}{*}{ dds } & \multicolumn{3}{|c|}{ Tasa absoluta de crecimiento $\left(g \cdot d i a^{-1}\right)$} \\
\hline & $\mathrm{T} 1$ & $\mathrm{~T} 2$ & T3 \\
\hline 9 & 0,0049 a & $0,0075 \mathrm{a}$ & 0,0111 b \\
\hline 12 & $0,0090 \mathrm{a}$ & $0,0130 \mathrm{~b}$ & $0,0177 \mathrm{c}$ \\
\hline 15 & $0,0135 \mathrm{a}$ & $0,0174 \mathrm{a}$ & $0,0225 \mathrm{~b}$ \\
\hline 18 & $0,0153 \mathrm{a}$ & $0,0169 \mathrm{a}$ & $0,0212 b$ \\
\hline 21 & $0,0135 \mathrm{a}$ & $0,0134 \mathrm{a}$ & $0,0168 \mathrm{~b}$ \\
\hline 24 & 0,0089 a & $0,0079 \mathrm{a}$ & $0,0102 \mathrm{~b}$ \\
\hline \multirow{2}{*}{ dds } & \multicolumn{3}{|c|}{ Tasa relativa de crecimiento $\left(\mathrm{g} \cdot \mathrm{g}^{-1} \cdot \mathrm{dí}^{-1}\right)$} \\
\hline & $\mathrm{T} 1$ & $\mathrm{~T} 2$ & T3 \\
\hline 9 & $0,234 \mathrm{a}$ & $0,220 \mathrm{~b}$ & $0,210 \mathrm{~b}$ \\
\hline 12 & 0,211 a & $0,192 b$ & $0,180 \mathrm{~b}$ \\
\hline 15 & $0,172 \mathrm{a}$ & $0,148 \mathrm{~b}$ & $0,137 \mathrm{~b}$ \\
\hline 18 & $0,121 \mathrm{a}$ & $0,097 \mathrm{~b}$ & $0,090 \mathrm{~b}$ \\
\hline 21 & $0,084 \mathrm{a}$ & $0,064 \mathrm{~b}$ & $0,060 \mathrm{~b}$ \\
\hline 24 & $0,045 \mathrm{a}$ & $0,033 \mathrm{~b}$ & $0,032 \mathrm{~b}$ \\
\hline \multirow{2}{*}{ dds } & \multicolumn{3}{|c|}{ Tasa de asimilación neta $\left(\mathrm{g} \cdot \mathrm{cm}^{-2} \cdot \mathrm{día}^{-1}\right)$} \\
\hline & $\mathrm{T} 1$ & $\mathrm{~T} 2$ & $\mathrm{~T} 3$ \\
\hline 9 & 0,00123 a & $0,00096 \mathrm{~b}$ & 0,00107 c \\
\hline 12 & $0,00092 \mathrm{a}$ & $0,00074 \mathrm{~b}$ & $0,00070 \mathrm{~b}$ \\
\hline 15 & 0,00065 a & $0,00053 \mathrm{~b}$ & $0,00048 \mathrm{~b}$ \\
\hline 18 & $0,00042 \mathrm{a}$ & $0,00034 \mathrm{~b}$ & $0,00033 \mathrm{~b}$ \\
\hline 21 & $0,00028 \mathrm{a}$ & 0,00023 a & $0,00024 \mathrm{a}$ \\
\hline 24 & $0,00015 \mathrm{a}$ & $0,00012 \mathrm{a}$ & $0,00013 \mathrm{a}$ \\
\hline
\end{tabular}

dds: días después de la siembra; T1 (Testigo), T2 (semillas lavadas con agua a TA) y T3: (semillas remojadas en agua a TA durante 24 horas). Valores con la misma letra dentro de tratamientos en cada hilera son iguales (Tukey; $\mathrm{p} \leq 0,05$ ).

Con relación a la TAN, para todos los tratamientos, su comportamiento inicial desde un valor máximo, descendió a medida que se dio el proceso de crecimiento del cultivo en fase de semillero (Figura 6).
Para el caso del tratamiento uno, la TAN superó con diferencias estadísticas significativas al resto de tratamientos en el periodo comprendido entre los 9 y 18 días después de la siembra (Tabla 4), lo cual corrobora que en las primeras etapas del cultivo la relación entre la acumulación de materia seca total y el área foliar es lineal, lo que no ocurre en las etapas tardías del proceso de crecimiento, como se observó desde los 19 días en adelante, que fisiológicamente se explica en términos, de que la tasa de crecimiento de área foliar puede exceder a la tasa de acumulación de materia seca o viceversa (Hunt, 1982 y Zhao et al., 2009).

Por su parte, la gráfica de AFE presentó una distribución normal (Figura 7). Los tratamientos uno y dos tuvieron valores más altos con respecto al tratamiento tres (Tabla 5), lo que posiblemente está asociado a respuestas morfológicas y fisiológicas de la planta por efecto de los tratamientos de remojo aplicados, lo que se puede manifestar en mejor grosor relativo o densidad del cánopi, lo cual confiere la ventaja de menor fragilidad foliar, con la mayor consecuente eficiencia fotosintética (Pérez et al., 2004; Días y De Carvalho, 2000).

Se ha encontrado que el AFE, favorece cambios en la RAF y la eficiencia fotosintética (Hunt, 1982; Gifford et al., 1984; Challa y Heuvelink, 1993 y Gardner et al., 1990). Lo anterior, pudo evidenciarse en la trayectoria gráfica de la RAF (Figura8), que mostró un comportamiento similar al AFE y que desde las fases iniciales del cultivo tuvieron para el tratamiento tres un incremento más sostenido y de más amplia distribución hasta alcanzar su máximo valor con respecto a los demás tratamientos a los 15 dds (Tabla 5). 
El IAF presentó una trayectoria gráfica simple sigmoide (Figura 9), encontrada también por Crisan et al. (2008), y que puede explicar la relación existente entre la superficie foliar expuesta a la radiación solar y el área de terreno ocupada por el cultivo y es reflejo de cómo fue la capacidad

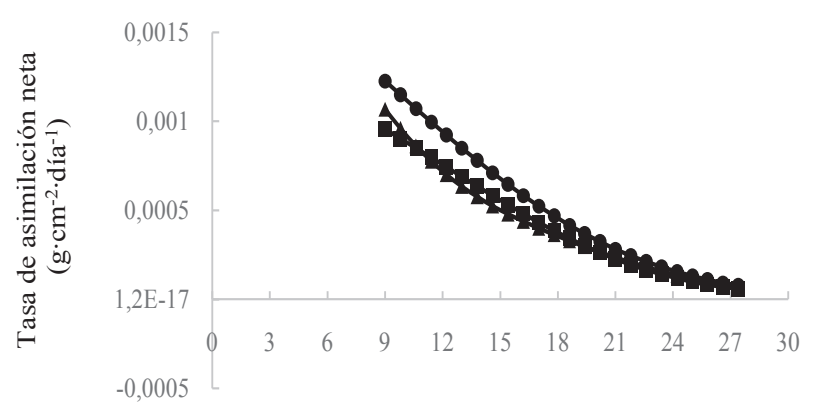

Días después de la siembra

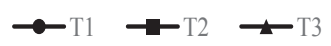

Figura 6. Tasa de asimilación neta de M. charantia en fase de semillero. T1 (Testigo), T2 (semillas lavadas con agua a TA) y T3: (semillas remojadas en agua a TA durante 24 horas). Montería, Colombia, 2014.

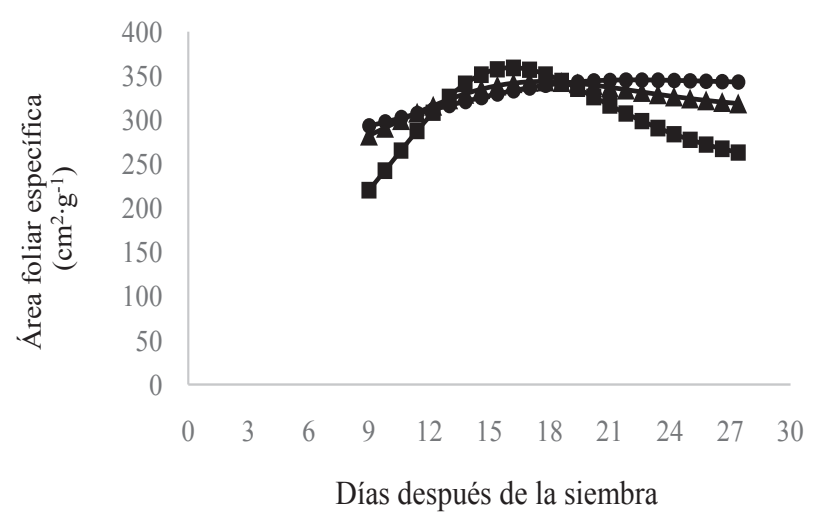

Figura 8. Relación de área foliar de M. charantia en fase de semillero. T1 (Testigo), T2 (semillas lavadas con agua a TA) y T3: (semillas remojadas en agua a TA durante 24 horas). Montería, Colombia, 2014. asimilatoria del sistema fotosintético de las plantas con relación a los tratamientos aplicados (Gardner et al., 1990). Para el caso del tratamiento tres, éste superó con diferencias estadísticas significativas a los demás tratamientos desde los 15 dds en adelante (Tabla 5).

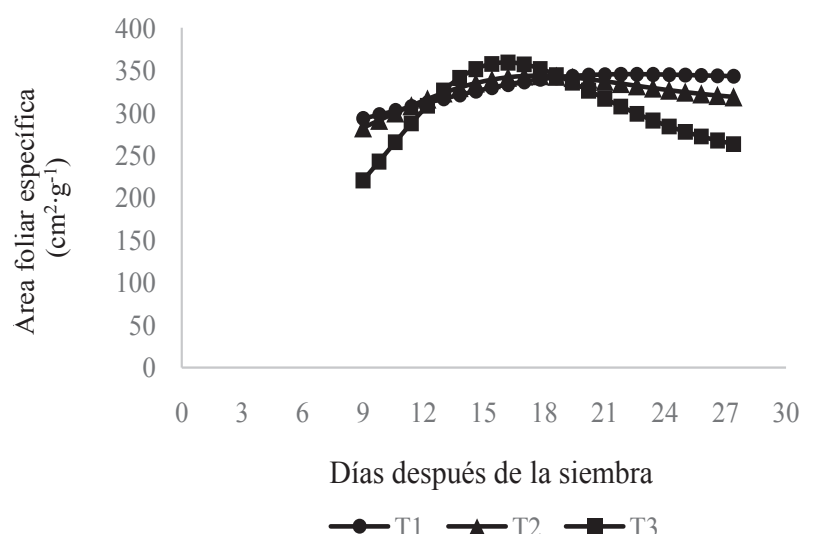

Figura 7. Área foliar específica de M. charantia en fase de semillero. T1 (Testigo), T2 (semillas lavadas con agua a TA) y T3: (semillas remojadas en agua a TA durante 24 horas). Montería, Colombia, 2014.

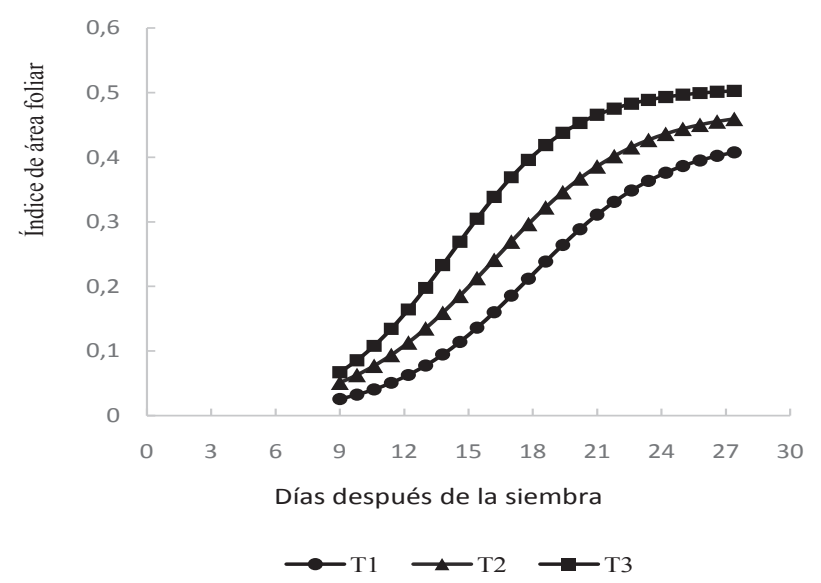

Figura 9. Índice de área foliar de M charantia en fase de semillero. T1 (Testigo), T2 (semillas lavadas con agua a TA) y T3: (semillas remojadas en agua a TA durante 24 horas). Montería, Colombia, 2014. 
Tabla 5. Área foliar específica, relación de área foliar, índice de área foliar y relación de peso foliar de $M$. charantia en fase de semillero, Montería, Colombia, 2014.

\begin{tabular}{|c|c|c|c|}
\hline \multirow{2}{*}{ dds } & \multicolumn{3}{|c|}{ Área foliar específica $\left(\mathrm{cm}^{2} \cdot \mathrm{g}^{-1}\right)$} \\
\hline & $\mathrm{T} 1$ & $\mathrm{~T} 2$ & $\mathrm{~T} 3$ \\
\hline 9 & $293,28 \mathrm{a}$ & $282,35 \mathrm{a}$ & $220,21 \mathrm{~b}$ \\
\hline 12 & $311,87 \mathrm{a}$ & $316,25 \mathrm{a}$ & $308,23 \mathrm{~b}$ \\
\hline 15 & $329,56 \mathrm{a}$ & 339,07 a & $357,64 \mathrm{~b}$ \\
\hline 18 & $341,51 \mathrm{a}$ & 343,10 a & $344,32 \mathrm{a}$ \\
\hline 21 & 344,98 a & 337,17 a & $316,56 \mathrm{a}$ \\
\hline 24 & $344,78 \mathrm{a}$ & $326,71 \mathrm{a}$ & $283,85 \mathrm{~b}$ \\
\hline \multirow{2}{*}{ dds } & \multicolumn{3}{|c|}{ Relación de área foliar $\left(\mathrm{cm}^{2} \cdot \mathrm{g}^{-1}\right)$} \\
\hline & $\mathrm{T} 1$ & $\mathrm{~T} 2$ & $\mathrm{~T} 3$ \\
\hline 9 & $293,28 \mathrm{a}$ & $282,35 \mathrm{a}$ & $220,21 \mathrm{~b}$ \\
\hline 12 & $311,87 \mathrm{a}$ & $316,25 \mathrm{a}$ & $308,23 \mathrm{~b}$ \\
\hline 15 & $329,56 \mathrm{a}$ & 339,07 a & $357,64 \mathrm{~b}$ \\
\hline 18 & $341,51 \mathrm{a}$ & 343,10 a & $344,32 \mathrm{a}$ \\
\hline 21 & $344,98 \mathrm{a}$ & 337,17 a & $316,56 \mathrm{~b}$ \\
\hline 24 & $344,78 \mathrm{a}$ & $326,71 \mathrm{a}$ & $283,85 \mathrm{~b}$ \\
\hline \multirow{2}{*}{ dds } & \multicolumn{3}{|c|}{ Índice de área foliar } \\
\hline & $\mathrm{T} 1$ & $\mathrm{~T} 2$ & $\mathrm{~T} 3$ \\
\hline 9 & $0,0258 \mathrm{a}$ & $0,0512 \mathrm{~b}$ & $0,0672 \mathrm{~b}$ \\
\hline 12 & $0,0631 \mathrm{a}$ & $0,1133 \mathrm{~b}$ & $0,1645 \mathrm{~b}$ \\
\hline 15 & $0,1361 \mathrm{a}$ & $0,2130 \mathrm{~b}$ & $0,3048 \mathrm{c}$ \\
\hline 18 & $0,2385 \mathrm{a}$ & $0,3223 \mathrm{~b}$ & $0,4186 \mathrm{c}$ \\
\hline 21 & $0,3109 \mathrm{a}$ & $0,3856 \mathrm{~b}$ & $0,4654 \mathrm{c}$ \\
\hline 24 & $0,3759 \mathrm{a}$ & $0,4360 \mathrm{~b}$ & $0,4930 \mathrm{c}$ \\
\hline \multirow{2}{*}{ dds } & \multicolumn{3}{|c|}{ Relación de peso foliar (\%) } \\
\hline & $\mathrm{T} 1$ & $\mathrm{~T} 2$ & $\mathrm{~T} 3$ \\
\hline 9 & $0,650 \mathrm{a}$ & $0,817 \mathrm{~b}$ & $0,892 \mathrm{~b}$ \\
\hline 12 & $0,732 \mathrm{a}$ & $0,817 \mathrm{~b}$ & $0,833 \mathrm{~b}$ \\
\hline 15 & $0,807 \mathrm{a}$ & $0,822 \mathrm{a}$ & 0,799 a \\
\hline 18 & $0,835 \mathrm{a}$ & $0,826 \mathrm{a}$ & 0,793 a \\
\hline 21 & $0,861 \mathrm{a}$ & $0,837 \mathrm{a}$ & $0,803 \mathrm{~b}$ \\
\hline 24 & $0,851 \mathrm{a}$ & $0,844 \mathrm{a}$ & $0,823 \mathrm{~b}$ \\
\hline
\end{tabular}

dds: días después de la siembra; T1 (Testigo), T2 (semillas lavadas con agua a TA) y T3 (semillas remojadas en agua a TA durante 24 horas). Valores con la misma letra dentro de tratamientos en cada hilera son iguales de acuerdo con la prueba de Tukey $(\mathrm{p} \leq 0,05)$.
Junto con el IAF, la RPF permite explicar porqué las plantas del tratamiento Tres, con respecto a las de los demás tratamientos, desde sus primeras etapas (Figura 10) exhibieron en cierta medida la mayor capacidad fisiológica de producción de materia seca (Tabla 5), que puede estar relacionada con la funcionalidad real de las hojas $\mathrm{u}$ órganos potencialmente fotosintéticos (Gardner et al., 1990), lo que se manifiesta esencialmente en una mayor cantidad y disponibilidad de fotoasimilados para el cultivo, para un crecimiento más vigoroso, importante en la fase de semillero para asegurar un buen trasplante a sitio definitivo y en lo sucesivo aumentar el rendimiento y calidad del cultivo tanto para su uso medicinal como en industria, siembra de cultivos y mejoramiento genético, entre otros (Gölükcü et al., 2014).

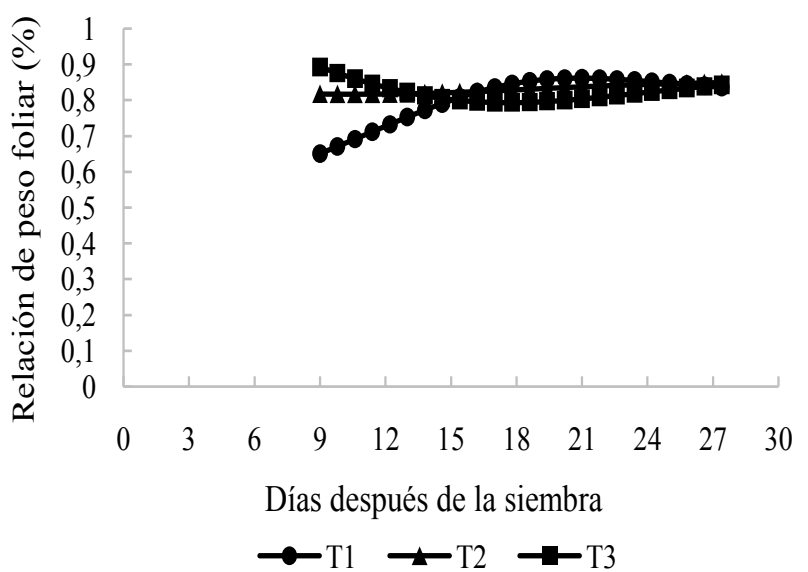

Figura 10. Relación de peso foliar de M. charantia en fase de semillero. T1 (Testigo), T2 (semillas lavadas con agua a TA) y T3: (semillas remojadas en agua a TA durante 24 horas). Montería, Colombia, 2014.

Respecto a los tratamientos aplicados, el comportamiento descrito a través del análisis de crecimiento, para la mayor acumulación de materia seca por fotosíntesis se basa en que el remojo de semillas en agua provee superior germinación, emergencia y establecimiento del cultivo y puede 
contribuir al crecimiento vigoroso en las primeras etapas, como es el caso del semillero, además de temprana floración, madurez y aumento en el rendimiento (Saleem et al., 2014 y Gölükcü et al., 2014).

\section{CONCLUSIONES}

La expresión cuantitativa del área foliar, materia seca de hojas y materia seca total de las plántulas de $M$. charantia en fase de semillero, fue mayor con el tratamiento de remojo de semillas en agua temperatura ambiente durante 24 horas.

El análisis de crecimiento efectuado a través de medidas indirectas mostró mayor tasa de incremento en acumulación de materia seca por unidad de tiempo (TAC) para las plántulas del tratamiento de remojo de semillas en agua a temperatura ambiente durante 24 horas.

El AFE y la RAF presentaron distribución normal a través del tiempo, con valores máximos a los 15 dds. Con el tratamiento de remojo de semillas con agua del grifo durante 24 horas, dichos valores fueron de mayor magnitud con respecto a los tratamientos testigo y semillas lavadas con agua.

El IAF presentó mayores valores durante el ciclo de semillero para las plántulas del tratamiento de remojo de semillas con agua del grifo durante 24 horas, lo que muestra que el crecimiento fue más rápido y de mayor magnitud con respecto a los tratamientos testigo y semillas lavadas con agua.

\section{REFERENCIAS}

ARDILA, G., FISCHER, G. y BALAGUERA-LÓPEZ, H. 2011. Caracterización del crecimiento del fruto y producción de tres híbridos de tomate (Solanum lycopersicum L.) en tiempo fisiológico bajo invernadero. Revista Colombiana de Ciencias Hortícolas. 5(1):44 - 56.

ASHRAFUZZAMAN, M., ISMAIL, M., ABDULLAH, K., UDDIN, M. y PRODHAN, A.2010. Effect of GABA application on the growth and yield of bitter gourd (Momordica charantia L.). International Journal of Agricultural and Biological Engineering. (12):129 - 132.

BÁEZ, M., TIJERINA, L., SÁNCHEZ, P., MARTÍNEZ, A., ACEVES, L. y ESCALANTE, J. 2002. Modelo logístico: herramienta para diagnosticar el cuánto y cuándo fertirrigar. Terra Latinoamericana. 21(2): 225 - 231.

BARRAZA, F. 2013. Crecimiento y calidad morfológica de berenjena (Solanum melongena L.) en fase de semillero. Temas Agrarios. 18(2):7 - 20.

BARRAZA, F. 2015. Physical characteristics and germination of bitter gourd (Momordica charantia Linn.) seeds. Indian Journal of Research. 4(5):3 - 5.

BEHERA, T., SINGH, A. y STAUB, J. 2008. Comparative analysis of genetic diversity in indian bitter gourd (Momordica charantia L.) using RAPD and ISSR markers for developing crop improvement strategies. Sciencia Horticulturae. 115:209 - 217.

BORREGO, F., FERNÁNDEZ, J., LÓPEZ, A., PARGA, V., MURILLO, M. y CARVAJAL, A. 2000. Análisis de crecimiento en siete variedades de papa (Solanum tuberosum L.). Agronomía Mesoamericana. 1(11):145 -149 .

CALVO, R., GONZÁLEZ, J. y PÉREZ, S. 1994. Manual de modelos no lineales en los ámbitos agronómico, ganadero y forestal. Primera edición. Ministerio de Agricultura, Pesca y Alimentación. Instituto Nacional de Investigación y Tecnología Agraria y Alimentaria. Madrid. 110 p.

CAUSTON, D. 1994. Plant growth analysis: a note on the variability of unit leaf rate (net assimilation rate) within a sample. Annals of Botany. 74:513 518. 
CHALLA, H. y HEUVELINK, E. 1993. Economic evaluation of crop photo synthesis. Acta Horticulturae. 328:219 - 228 .

CLAVIJO, J. 1989. Análisis del crecimiento en malezas. Revista COMALFI. 16 (12 - 16): 13 - 16.

CRIOLLO, H. y GARCÍA, J. 2009. Efecto de la densidad de siembra sobre el crecimiento de plantas de rábano (Raphanus satious L.) bajo invernadero. Revista Colombiana de Ciencias Hortícolas. 3(2):210 $-222$.

CRISAN, S., CAMPEANU, G. y HALMAGEAN, L. 2008. Researches regarding fertilization'sin fluence on the yield of unripe fruits for Momordic acharantia L. species, on different soiltypes in arad agroecological area. Bulletin of University of Agricultural Sciences and Veterinary Medicine Cluj-Napoca. Horticulture. 65(1):368 -373.

DÍAS, M. y DE CARVALHO, C. 2000. Physiological and morphological responses of Brachiaria spp. to flooding. Pesquisa Agropecuária Brasileira. 35(10):1959 - 1966.

EVANS, G. 1972. The quantitative analysis of plant growth. First edition. Blackwell Scientific Publications, Oxford. 733 p.

FERREIRA, O., ROSSI, F. y ANDRIGHETTO, C. 2009.Determinação de área foliar, índice de área foliar e área de olho de lombo através de imagens digitais. p. 1-3. En: Memorias 46a Reunião Anual da Sociedade Brasileira de Zootecna. Maringá, Brasil.

FONNEGRA, R. y JIMÉNEZ, S. 2007. Plantas medicinales aprobadas en Colombia. Segunda Edición, Editorial Universidad de Antioquia. Medellín. 371 p.

FOHS, A., KREJPCIO, Z., KRÓL, E., XIONG, R. y HOŁUBOWICZ, R. 2014. The effect of cultivation ways on selected morphological characters of bitter gourd (Momordica charantia L.) transplants and plants, fruit yield and chemical content. Bulletin of University of Agricultural Sciences and Veterinary Medicine Cluj-Napoca. Horticulture.71(1):38 - 42.
GARDNER, F., PEARCE, R. y MITCHELL, R. 1990. Physiology of crop plants. First edition, Iowa University Press, Ames.327 p.

GIFFORD, R., THORNE, J., HITZ, W. y GIAQUINTA, R. 1984. Crop productivity and photo as similate partitioning. Science. 225(4644):801 - 808.

GÖLÜKCÜ, M., TOKER, R., AYAS, F. y ÇINAR, N. 2014.Some physical and chemical properties of bitter melon (Momordica charantia L.) seed and fatty acid composition of seed oil. Anadolu University Journal of Science and Technology. 31(1):17 - 24.

HARRIS, D. 2003. Increasing yields of rainfed crops in Africa using'on-farm' seed priming. African Crop Science Conference Proceedings. 6:26 - 30.

HUNT, R. 1982. Plant growth analysis: second derivatives and compounded second derivatives of splined plant growth curves. Annals of Botany. 50(3):317 - 328.

HUNT, R. 1990. Basic growth analysis: plant growth analysis for beginners. First edition. Unwin Hyman Ltd., Londres. 112 p.

HUNT, R., CAUSTON, D., SHIPLEY, B. y ASKEW, P. 2002. A Modern Tool for classical plant growth analysis. Annals of Botany. 90:485 - 488.

KUO, C., LEE, T.y TSAI, P. 2012. Morphological and physiological changes of bitter gourd (Momordica charantia L., cv. Moom Shine during fruit development. Journal of Ilan Institute of Technology. 3:25 - 34.

MAHAJAN, T. y PANDEY, O. 2015. Effect of electric and magnetic treatments on germination of bitter gourd (Momordica charantia) seed. International Journal of Agriculture and Biology. 17(2):351 -356.

MIA, B., ISLAM, S., MIAH, Y., DAS, M. y KHAN, H. 2014. Flower synchrony, growth and yield enhancement of small type bitter gourd (Momordica charantia L.) through plant growth regulators and NPK fertilization. Pakistan Journal of Biological Sciences 17 (3): 408 - 413. 
MIAN, M., MOROKUMA, M., ALI, M., AGATA, W. y FUJEIDA, K. 1993. Effect of intergeneric grafting on growth and photosynthesis in Momordica dioica Roxb. Journal of the Faculty of Agriculture. 38(1-2):65 - 71.

PÉREZ-ARBELÁEZ, E.1994. Plantas útiles de Colombia. Decimocuarta edición, Editorial Víctor Hugo, Medellín. 832 p.

PÉREZ, J., GARCÍA, E., ENRÍQUEZ, J., QUERO, A., PÉREZ, J. y HERNÁNDEZ, A. 2004. Análisis de crecimiento, área foliar específica y concentración de nitrógeno en hojas de pasto "mulato" (Brachiaria híbrido, cv.). Revista Mexicana de Ciencias Pecuarias. 42(3):447 - 458.

POORTER, H. 1989. Plant growth analysis: towards a synthesis of the classical and the functional approach. Physiologia Plantarum. 75: 237 - 244.

PRUSINKIEWICZ, P. 2004. Modeling plant growth and development. Current Opinion in Plant Biology. 7(1):79 - 83.

SALEEM, M., SAJID, M., AHMED, Z., AHMED, S., AHMED, N. e ISLAM, M. 2014. Effect of seed soaking on seed germination and growth of bitter gourd cultivars. Journal of Agriculture and Veterinary Science. 6(6):7 - 11.

SAS INSTITUTE INC. 2008. Statistical analysis system. The $\mathrm{SAS}^{\oplus}$ system for Windows ${ }^{\odot}$ version 9.1.3. The Power to Know. Cary, NC, USA.

ŞAVŞATLIY, Y. y SEYIS, F. 2014. Effects of different planting frequency to some agricultural traits of bitter melón (Momordica charantia L.) grown in Rize ecological conditions. Turkish Journal of Agricultural and Natural Sciences. 1:659 - 662.

SHAHADAT, H., MOSTOFA, M., MAMUN, A., HOQUE, E. y AWAL, M. 2008. Comparative efficacy of corolla (Momordica charantia) extract and Ivermec $^{\circledR}$ pour on with their effects on certain blood parameters and body weight gain in indigenous chicken infected with Ascaridiagalli. Bangl. The Journal of Veterinary Medical Science. 6(2):153 - 158.
THAKUR, G., BAG, M., SANODIYA, B., BHADAURIYA, P., DEBNATH, M., PRASAD, G. y BISEN, P. 2009. Momordica balsamina: a medicinal and neutraceutical plant for health care management. Current Pharmaceutical Biotechnology. 10:667 - 682.

WANG, X., OU-YANG, CH., FAN, Z., GAO, S., CHEN, F. y TANG, L. 2010. Effects of exogenous silicon on seed germination and antioxidant enzyme activities of Momordicacharantia under salt stress. Journal of Animal and Plant Sciences. 6(3):700 - 708.

ZHAO, D., MA, Z., LI, Z. y XIAO, Y. 2009. Growth and photosynthetic capability of Momordica grosvenori plantlets grown photo autotrophically in response to light intensity. Horticultural Science. 44(3):757 - 763.

ZONG, R., MORRIS, L. y CANTWELL, M. 1995. Postharvest physiology and quality of bitter melon (Momordica charantia L.). Postharvest Biology and Technology. 6:65 - 72 . 